\title{
The Poincaré Series of Some Special Quasihomogeneous Surface Singularities
}

By

Wolfgang EBELING*

\begin{abstract}
In [E6] a relation is proved between the Poincaré series of the coordinate algebra of a two-dimensional quasihomogeneous isolated hypersurface singularity and the characteristic polynomial of its monodromy operator. We study this relation for Fuchsian singularities and show that it is connected with the mirror symmetry of K3 surfaces and with automorphisms of the Leech lattice. We also indicate relations between other singularities and Conway's group.
\end{abstract}

\section{Introduction}

K. Saito [Sa1], [Sa2] has introduced a duality between polynomials which are products of cyclotomic polynomials. He has shown that V. I. Arnold's strange duality between the 14 exceptional unimodal hypersurface singularities is related to such a duality between the characteristic polynomials of the monodromy operators of the singularities. Moreover, he has observed that the dual polynomials pair together to the characteristic polynomial of an automorphism of the Leech lattice. It is now well-known that Arnold's strange duality is related to the mirror symmetry of $K 3$ surfaces (see e.g. [D5]).

The author [E4], [E5] has shown that these features still hold in a certain way for the extension of Arnold's strange duality discovered by C. T. C. Wall and the author $[\mathrm{EW}]$.

Communicated by K. Saito. Received October 5, 2001.

2000 Mathematics Subject Classification(s): Primary 14J17, 32S25, 32S40, 13D40; Secondary 14J28, $11 \mathrm{H} 56$.

Partially supported by the DFG-programme "Global methods in complex geometry" (Eb 102/4-1).

*Institut für Mathematik, Universität Hannover, Postfach 6009, D-30060 Hannover, Germany.

e-mail: ebeling@math.uni-hannover.de 
The 14 exceptional singularities and the singularities involved in the extension of Arnold's strange duality are examples of Fuchsian singularities. By this we mean the following. Let $\Gamma \subset \operatorname{PSL}(2, \mathbb{R})$ be a finitely generated Fuchsian group of the first kind. Let $A_{k}$ denote the $\mathbb{C}$-vector space of $\Gamma$-automorphic forms of weight $2 k, k \geq 0$, and let $A=\bigoplus_{k=0}^{\infty} A_{k}$ be the algebra of $\Gamma$ automorphic forms. Then $(X, x):=(\operatorname{Spec} A, \mathfrak{m})$ where $\mathfrak{m}:=\bigoplus_{k=1}^{\infty} A_{k}$ is a normal surface singularity which is called a Fuchsian singularity.

In this paper we show that the observation of K. Saito extends in a certain sense to all Fuchsian singularities which are isolated complete intersection singularities (abbreviated ICIS in the sequel), although for some of the polynomials there is a singularity missing.

The basis of this duality forms a relation between the Poincaré series of the coordinate algebra of such a singularity and the characteristic polynomial of its monodromy operator which was considered in [E6]. There we introduced polynomials $\phi_{A}(t)$ and $\psi_{A}(t)$ as follows. Let $(X, x)$ be a normal surface singularity with good $\mathbb{C}^{*}$-action. The coordinate algebra $A$ is a graded algebra. We consider the Poincaré series $p_{A}(t)$ of $A$. Let $\left\{g ; b ;\left(\alpha_{1}, \beta_{1}\right), \ldots,\left(\alpha_{r}, \beta_{r}\right)\right\}$ be the orbit invariants of $(X, x)$. We define

$$
\begin{aligned}
& \psi_{A}(t):=(1-t)^{2-r} \prod_{i=1}^{r}\left(1-t^{\alpha_{i}}\right), \\
& \phi_{A}(t):=p_{A}(t) \psi_{A}(t)
\end{aligned}
$$

If $(X, x)$ is a Fuchsian singularity, then we show that the polynomial $\phi_{A}(t)$ can be interpreted as the characteristic polynomial of a certain Coxeter element $c_{\infty}$ (Proposition 1). In the case of Arnold's strange duality, the Coxeter element $c_{\infty}$ is the monodromy operator of the dual singularity.

If $(X, x)$ is a Fuchsian ICIS, then we derive from the results of [E6] that we can slightly modify the polynomial $\phi_{A}(t)$ to a rational function $\tilde{\phi}_{A}(t)$ such that the dual (in Saito's sense) of $\tilde{\phi}_{A}(t)$ is the characteristic polynomial $\phi_{M}(t)$ of the monodromy operator of $(X, x)$ (or a slightly modified polynomial $\phi_{M}^{b}(t)$ ). We relate this duality to the mirror symmetry of K3 surfaces.

We show that for a Fuchsian ICIS the rational function $\tilde{\phi}_{A}(t)$ and its dual pair together to the characteristic polynomial of an automorphism of the Leech lattice. Moreover, we consider the quasihomogeneous hypersurface singularities in $\mathbb{C}^{3}$ with Milnor number $\mu=24$. It was already observed by K. Saito that the polynomials $\phi_{M}(t)$ of these singularities are self-dual and are also characteristic polynomials of automorphisms of the Leech lattice. We show that the same is true for the polynomials $\phi_{M}^{b}(t)$ of some ICIS in $\mathbb{C}^{4}$ with $\mu=25$. Finally we 
indicate 5 constructions which lead from singularities to self-dual characteristic polynomials of automorphisms of the Leech lattice and we show that all such polynomials can be obtained in a suitable way.

The paper is organized as follows. In Section 1 we recall the structure of normal surface singularities with good $\mathbb{C}^{*}$-action and the definition of Fuchsian singularities. In Section 2 we review the relevant results of [E6] about the relation between Poincaré series and monodromy. In Section 3 we consider the polynomial $\phi_{A}(t)$ of a Fuchsian singularity and show that it is the characteristic polynomial of a Coxeter element $c_{\infty}$. In Section 4 we derive the duality among the Fuchsian ICIS and relate it to the mirror symmetry of K3 surfaces. Finally we discuss the relation to automorphisms of the Leech lattice in Section 5.

The author thanks R.-O. Buchweitz for pointing out an error in an earlier version of the paper and C. T. C. Wall for pointing out that the list of ICIS in $\mathbb{C}^{4}$ with $\mu=25$ in that version was incomplete. He is grateful to the referee for his useful comments.

\section{$\S 1$ Quasihomogeneous Surface Singularities}

Let $(X, x)$ be a normal surface singularity with a good $\mathbb{C}^{*}$-action. So $X$ is a normal two-dimensional affine algebraic variety over $\mathbb{C}$ which is smooth outside its vertex $x$. Its coordinate ring $A$ has the structure of a graded $\mathbb{C}$-algebra $A=\bigoplus_{k=0}^{\infty} A_{k}, A_{0}=\mathbb{C}$, and $x$ is defined by the maximal ideal $\mathfrak{m}=\bigoplus_{k=1}^{\infty} A_{k}$.

According to I. Dolgachev [D2], there exist a simply connected Riemann surface $\mathcal{D}$, a discrete cocompact subgroup $\Gamma$ of $\operatorname{Aut}(\mathcal{D})$ and a line bundle $\mathcal{L}$ on $\mathcal{D}$ to which the action of $\Gamma$ lifts such that

$$
A_{k}=H^{0}\left(\mathcal{D}, \mathcal{L}^{k}\right)^{\Gamma}
$$

Let $Z:=\mathcal{D} / \Gamma$. By [P2, Theorem 5.1] (see also [Wag2, Theorem 5.4.1]), there exist a divisor $D_{0}$ on $Z, p_{1}, \ldots, p_{r} \in Z$, and integers $\alpha_{i}, \beta_{i}$ with $0<\beta_{i}<$ $\alpha_{i}$ and $\left(\alpha_{i}, \beta_{i}\right)=1$ for $i=1, \ldots, r$ such that

$$
A_{k}=L\left(k D_{0}+\sum_{i=1}^{r}\left[k \frac{\alpha_{i}-\beta_{i}}{\alpha_{i}}\right] p_{i}\right) .
$$

Here $[x]$ denotes the largest integer $\leq x$, and $L(D)$ for a divisor $D$ on $Z$ denotes the linear space of meromorphic functions $f$ on $Z$ such that $(f) \geq-D$. We number the points $p_{i}$ so that $\alpha_{1} \leq \alpha_{2} \leq \cdots \leq \alpha_{r}$. Let $g$ be the genus of $Z$ and define $b:=\operatorname{degree} D_{0}+r$. Then $\left\{g ; b ;\left(\alpha_{1}, \beta_{1}\right), \ldots,\left(\alpha_{r}, \beta_{r}\right)\right\}$ are called the orbit invariants of $(X, x)$, cf. e.g. [Wag3]. $\operatorname{Define} \operatorname{vdeg}(\mathcal{L}):=-b+\sum_{i=1}^{r} \frac{\beta_{i}}{\alpha_{i}}$. 
Now assume that $(X, x)$ is Gorenstein. By [D4], there exists an integer $R$ such that $\mathcal{L}^{-R}$ and the tangent bundle $T_{\mathcal{D}}$ of $\mathcal{D}$ are isomorphic as $\Gamma$-bundles and

$$
\begin{aligned}
R \cdot \operatorname{vdeg}(\mathcal{L}) & =2-2 g-r+\sum_{i=1}^{r} \frac{1}{\alpha_{i}}, \\
R \beta_{i} & \equiv 1 \bmod \alpha_{i}, \quad i=1, \ldots, r .
\end{aligned}
$$

Following [D3, 3.3.15] we call $R$ the exponent of $(X, x)$. Since $b$ and the $\beta_{i}$ are determined by the $\alpha_{i}$ and the number $R$, we write the orbit invariants also as $g ; \alpha_{1}, \ldots, \alpha_{r}$.

If $R=1$, then we have the following situation. In this case $\Gamma \subset P S L(2, \mathbb{R})$ is a finitely generated cocompact Fuchsian group of the first kind. This means that $\Gamma$ acts properly discontinuously on $\mathbb{H}$ and that the quotient $Z=\mathbb{H} / \Gamma$ is a compact Riemann surface. The divisor $D_{0}$ is the canonical divisor, the points $p_{1}, \ldots, p_{r} \in Z$ are the branch points of the map $\mathbb{H} \rightarrow Z, \alpha_{i}$ is the ramification index over $p_{i}$, and $\beta_{i}=1$ for $i=1, \ldots, r$. Hence the orbit invariants are

$$
\left\{g ; 2 g-2+r ;\left(\alpha_{1}, 1\right), \ldots,\left(\alpha_{r}, 1\right)\right\} .
$$

We follow [Lo] in calling $(X, x)$ a Fuchsian singularity. The orbit invariants $\left\{g ; \alpha_{1}, \ldots, \alpha_{r}\right\}$ are also called the signature of $\Gamma$.

\section{§2. The Poincaré Series}

Let $(X, x)$ be a normal surface singularity with good $\mathbb{C}^{*}$-action with orbit invariants $\left\{g ; b ;\left(\alpha_{1}, \beta_{1}\right), \ldots,\left(\alpha_{r}, \beta_{r}\right)\right\}$. Let $p_{A}(t)$ be the Poincaré series of the coordinate algebra $A$ of $(X, x)$. We define

$$
\begin{aligned}
& \psi_{A}(t):=(1-t)^{2-r} \prod_{i=1}^{r}\left(1-t^{\alpha_{i}}\right), \\
& \phi_{A}(t):=p_{A}(t) \psi_{A}(t) .
\end{aligned}
$$

Let $(X, x)$ be an ICIS with weights $q_{1}, \ldots, q_{n}$ and degrees $d_{1}, \ldots, d_{n-2}$. Then its Poincaré series is given by (see e.g. [Wag3, Proposition (2.2.2)])

$$
p_{A}(t)=\frac{\prod_{i=1}^{n-2}\left(1-t^{d_{i}}\right)}{\prod_{j=1}^{n}\left(1-t^{q_{j}}\right)} .
$$

Therefore $p_{A}(t), \psi_{A}(t)$ and $\phi_{A}(t)$ are rational functions of the form

$$
\phi(t)=\prod_{m \mid h}\left(1-t^{m}\right)^{\chi_{m}} \quad \text { for } \chi_{m} \in \mathbb{Z} \text { and for some } h \in \mathbb{N} .
$$


Given a rational function

$$
\phi(t)=\prod_{m \mid h}\left(1-t^{m}\right)^{\chi_{m}}
$$

K. Saito [Sa1] has defined a dual rational function

$$
\phi^{*}(t)=\prod_{k \mid h}\left(1-t^{k}\right)^{-\chi_{h / k}}
$$

In [E6] we proved the following results. For integers $a_{1}, \ldots, a_{r}$ we denote by $\left\langle a_{1}, \ldots, a_{r}\right\rangle$ their least common multiple and by $\left(a_{1}, \ldots, a_{r}\right)$ their greatest common divisor.

Theorem 1. Let $(X, x)$ be a quasihomogeneous hypersurface singularity in $\mathbb{C}^{3}$. Consider the rational function

$$
\tilde{\phi}_{A}(t):=\frac{\phi_{A}(t)}{(1-t)^{2 g}} .
$$

Then $\tilde{\phi}_{A}^{*}(t)$ is the characteristic polynomial of the classical monodromy operator of $(X, x)$.

Theorem 2. Let $(X, x)$ be a quasihomogeneous ICIS in $\mathbb{C}^{4}$ with weights $q_{1}, q_{2}, q_{3}, q_{4}$ and degrees $d_{1}, d_{2}$. Assume that $g\left(z_{1}, z_{2}, z_{3}, z_{4}\right)=z_{1} z_{4}+z_{2} z_{3}$. Define

$$
\tilde{\phi}_{A}(t):=\frac{\phi_{A}(t)\left(1-t^{d_{2}}\right)}{(1-t)^{2 g}\left(1-t^{d_{1}}\right)}, \quad \phi_{M}^{b}(t):=\frac{\phi_{M}(t)}{(1-t)} .
$$

Then we have $\tilde{\phi}_{A}^{*}(t)=\phi_{M}^{b}(t)$.

Theorem 3. Let $(X, x)$ be a quasihomogeneous ICIS in $\mathbb{C}^{4}$ with weights $q_{1}, q_{2}, q_{3}, q_{4}$ and degrees $d_{1}, d_{2}$. Assume that either

(A) $g\left(z_{1}, z_{2}, z_{3}, z_{4}\right)=z_{1}^{q}+z_{2} z_{3}$ and $f\left(z_{1}, z_{2}, z_{3}, z_{4}\right)=f^{\prime}\left(z_{1}, z_{2}, z_{3}\right)+z_{4}^{p}$ for some integers $p, q \geq 2$ where $q \mid d_{2}$, or

(B) $g\left(z_{1}, z_{2}, z_{3}, z_{4}\right)=z_{1}^{q}+\left(z_{2}-z_{3}\right) z_{4}$ and $f\left(z_{1}, z_{2}, z_{3}, z_{4}\right)=a z_{1}^{q}+z_{2}\left(z_{3}-z_{4}\right)$ for some $a \in \mathbb{C}, a \neq 0,1$, and some integer $q \geq 2$ and $p:=2$.

Define

$$
\begin{aligned}
\tilde{\phi}_{A}(t) & :=\frac{\phi_{A}(t)\left(1-t^{d_{2}}\right)^{p-1}\left(1-t^{\frac{d_{1}}{q}}\right)\left(1-t^{\frac{d_{2}}{p}}\right)}{(1-t)^{2 g}\left(1-t^{d_{1}}\right)\left(1-t^{\frac{d_{2}}{q}}\right)^{p}}, \\
\phi_{M}^{b}(t) & :=\frac{\phi_{M}(t)\left(1-t^{q}\right)^{p}}{(1-t)^{p-1}\left(1-t^{\langle p, q\rangle}\right)^{(p, q)}} .
\end{aligned}
$$

Then we have $\tilde{\phi}_{A}^{*}(t)=\phi_{M}^{b}(t)$. 
The polynomial $\phi_{M}^{b}(t)$ was already introduced for the singularities in the extension of Arnold's strange duality in [E4]. There one can also find some background for this construction.

For the proofs of these theorems, one compares the invariants on both sides of the corresponding equalities by using the known formulas and verifies that they coincide. For details see [E6]. Nevertheless, one would still like to understand whether there is a deeper reason for these rather mysterious relations between algebraic and topological invariants.

\section{$\S 3 . \quad$ The Poincaré Series of a Fuchsian Singularity}

Now let $(X, x)$ be a Fuchsian singularity. Let $p_{A}(t)$ be the Poincaré series of the algebra $A$. We have

$$
p_{A}(t)=\frac{1+(g-2) t+(g-2) t^{2}+t^{3}}{(1-t)^{2}}+\sum_{i=1}^{r} \frac{t^{2}\left(1-t^{\alpha_{i}-1}\right)}{(1-t)^{2}\left(1-t^{\alpha_{i}}\right)} .
$$

We shall now show that the polynomial $\phi_{A}(t)$ can also be interpreted as the characteristic polynomial of a certain operator.

Let $(X, x)$ be a normal surface singularity with good $\mathbb{C}^{*}$-action. Then $X$ can be compactified to $\bar{X}$ in a natural way (see [P2]). The variety $\bar{X}$ has $r$ cyclic quotient singularities of type $\left(\alpha_{1}, \alpha_{1}-\beta_{1}\right), \ldots,\left(\alpha_{r}, \alpha_{r}-\beta_{r}\right)$ along $\bar{X}_{\infty}:=\bar{X}-X$ [P2, Lemma 4.1].

Now assume that $(X, x)$ is Fuchsian. Then $\beta_{i}=1$ for all $i=1, \ldots, r$. A cyclic quotient singularity of type $(\alpha, \alpha-1)$ is a singular point of type $A_{\alpha-1}$. Let $\pi: \tilde{X} \rightarrow \bar{X}$ be the minimal resolution of the singularities of $\bar{X}$ along $\bar{X}_{\infty}$. The preimage $\tilde{X}_{\infty}$ of $\bar{X}_{\infty}$ consists of the strict transform $\varepsilon_{\infty}$ of $\bar{X}_{\infty}$ and $r$ chains $\delta_{i, 1}, \ldots, \delta_{i, \alpha_{i}-1}, i=1, \ldots, r$, of rational curves of self-intersection -2 which intersect according to the dual graph shown in Figure 1. By the adjunction formula, the self-intersection number of the curve $\varepsilon_{\infty}$ is $2 g-2$.

Let $M_{\infty}$ be the abstract lattice spanned by these curves, i.e., the free $\mathbb{Z}$ module spanned by $\delta_{1,1}, \ldots, \delta_{1, \alpha_{1}-1} ; \ldots ; \delta_{r, 1}, \ldots, \delta_{r, \alpha_{r}-1} ; \varepsilon_{\infty}$ with the bilinear form $\langle$,$\rangle given by the intersection numbers. Let U$ be a unimodular hyperbolic plane, i.e., a free $\mathbb{Z}$-module with basis $\left\{f_{1}, f_{2}\right\}$ satisfying $\left\langle f_{1}, f_{1}\right\rangle=\left\langle f_{2}, f_{2}\right\rangle=0$, $\left\langle f_{1}, f_{2}\right\rangle=\left\langle f_{2}, f_{1}\right\rangle=1$. We shall consider the lattice $N=M_{\infty} \oplus U$. Special automorphisms of this lattice are defined as follows. If $\delta \in N$ is a vector of squared length $\langle\delta, \delta\rangle= \pm 2$, then

$$
s_{\delta}(x)=x-\frac{2\langle x, \delta\rangle}{\langle\delta, \delta\rangle} x \quad \text { for } x \in N
$$




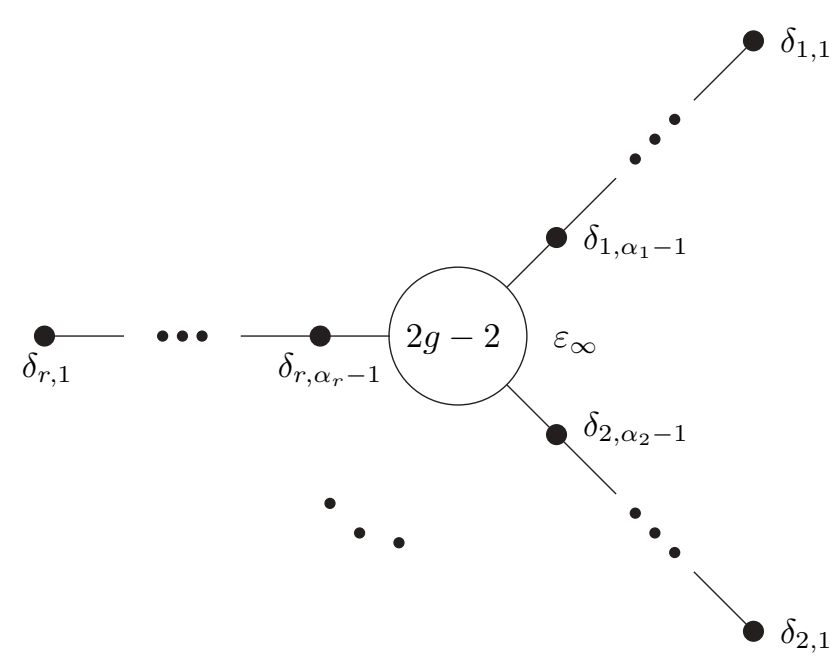

Figure 1. Dual graph of $\tilde{X}_{\infty}$.

defines the reflection $s_{\delta}$ corresponding to $\delta$. For $f \in U$ with $\langle f, f\rangle=0$ and $w \in$ $M_{\infty}$ we define a transformation $\psi_{f, w}$, called the Eichler-Siegel transformation corresponding to $f$ and $w$, by the formula

$$
\psi_{f, w}(x)=x+\langle x, f\rangle w-\langle x, w\rangle f-\frac{1}{2}\langle w, w\rangle\langle x, f\rangle f
$$

for $x \in N$ (cf. [E2]).

Proposition 1. Let $(X, x)$ be a Fuchsian singularity. Then the polynomial $\phi_{A}(t)$ is the characteristic polynomial of the operator

$$
c_{\infty}=s_{\delta_{1,1}} \cdots s_{\delta_{1, \alpha_{1}-1}} \cdots s_{\delta_{r, 1}} \cdots s_{\delta_{r, \alpha_{r}-1}} \psi_{f_{1}, \varepsilon_{\infty}} s_{f_{1}-f_{2}} .
$$

Proof. In order to compute the characteristic polynomial of $c_{\infty}$, we want to apply [B, Chapter V, Section 6, Exercice 3]. For two vectors $u, v \in N$ the pseudo-reflection $s_{u, v}$ is defined by

$$
s_{u, v}(x)=x-\langle x, v\rangle u \text {. }
$$

An easy calculation shows that the Eichler-Siegel transformation $\psi_{f, w}$ can be written as a product of two pseudo-reflections as follows:

$$
\psi_{f, w}=s_{\tilde{w}, f} s_{f, w} \quad \text { where } \tilde{w}:=\frac{1}{2}\langle w, w\rangle f-w .
$$


In the basis

$$
\varepsilon_{1}:=\frac{1}{2}\left\langle\varepsilon_{\infty}, \varepsilon_{\infty}\right\rangle f_{1}-\varepsilon_{\infty}, \quad \varepsilon_{2}:=f_{1}, \quad \varepsilon_{3}:=f_{1}-f_{2},
$$

the operator $\psi_{f_{1}, \varepsilon_{\infty}} s_{f_{1}-f_{2}}$ can be written as $s_{1} s_{2} s_{3}$ where

$$
s_{i}\left(\varepsilon_{j}\right)=\varepsilon_{j}-a_{i j} \varepsilon_{i}, \quad 1 \leq i, j \leq 3, \quad\left(a_{i j}\right)_{1 \leq j \leq 3}^{1 \leq i \leq 3}=\left(\begin{array}{ccc}
0 & 0 & -1 \\
-\left\langle\varepsilon_{\infty}, \varepsilon_{\infty}\right\rangle & 0 & 0 \\
\frac{1}{2}\left\langle\varepsilon_{\infty}, \varepsilon_{\infty}\right\rangle & 1 & 2
\end{array}\right) .
$$

By [B, loc.cit.] we get using $\left\langle\varepsilon_{\infty}, \varepsilon_{\infty}\right\rangle=2 g-2$

$$
\operatorname{det}\left(t I-s_{1} s_{2} s_{3}\right)=\left|\begin{array}{ccc}
t-1 & 0 & -t \\
2-2 g & t-1 & 0 \\
g-1 & 1 & t+1
\end{array}\right|=1+(g-2) t+(g-2) t^{2}+t^{3} .
$$

This proves Proposition 1 for the case $r=0$. The general case also follows by using the formula of [B, loc.cit.] for the determinant of the matrix $t I-c_{\infty}$ and the Laplace expansion formula.

If $g=0$, then we have $\left\langle\varepsilon_{\infty}, \varepsilon_{\infty}\right\rangle=-2$ and from [E2, Section 5.1, (c1)] we conclude that

$$
\psi_{f_{1}, \varepsilon_{\infty}}=s_{\varepsilon_{\infty}} \circ s_{\varepsilon_{\infty}-f_{1}}
$$

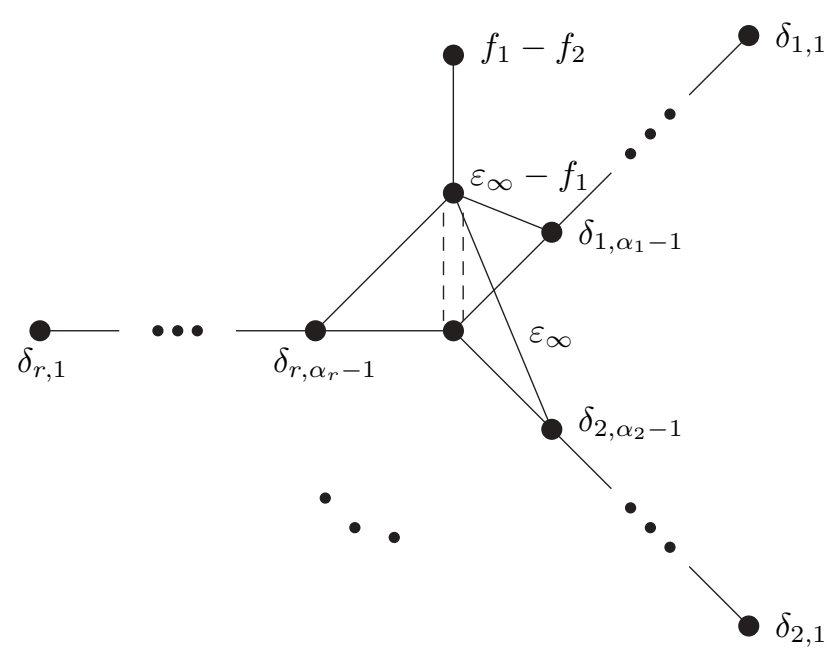

Figure 2. The graph $\tilde{\theta}_{\alpha_{1}, \ldots, \alpha_{r}}$. 
Therefore the operator $c_{\infty}$ coincides with the Coxeter element corresponding to the graph of Figure 2 and its characteristic polynomial is already given in [E2, Section 3.4] (unfortunately with a misprint).

Remark 1. Consider the polynomial

$$
\psi_{A}(t)=(1-t)^{2-r} \prod_{i=1}^{r}\left(1-t^{\alpha_{i}}\right)
$$

for integers $r \geq 1, \alpha_{i} \geq 1, i=1, \ldots, r$. By [E2, p. 98], this polynomial is the characteristic polynomial of the Coxeter element corresponding to the graph $\theta_{\alpha_{1}, \ldots, \alpha_{r}}$ which is obtained from the graph of Figure 2 by omitting the vertex corresponding to the vector $f_{1}-f_{2}$. The dual rational function is

$$
\psi_{A}^{*}(t)=\frac{\left(1-t^{\left\langle\alpha_{1}, \ldots, \alpha_{r}\right\rangle}\right)^{r-2}}{\prod_{i=1}^{r}\left(1-t^{\left\langle\alpha_{1}, \ldots, \alpha_{r}\right\rangle / \alpha_{i}}\right)} .
$$

For $r \geq 3$ this rational function is the Poincaré series of the Brieskorn-Hamm ICIS $\left(V_{B}\left(\alpha_{1}, \ldots, \alpha_{r}\right), 0\right)$ where

$$
V_{B}\left(\alpha_{1}, \ldots, \alpha_{r}\right):=\left\{z \in \mathbb{C}^{r} \mid b_{i 1} z_{1}^{\alpha_{1}}+\cdots+b_{i r} z_{r}^{\alpha_{r}}=0 ; i=1, \ldots, r-2\right\}
$$

and $B=\left(b_{i j}\right)$ is a sufficiently general $(r-2) \times r$-matrix of complex numbers. If $\left(V_{B}\left(\alpha_{1}, \ldots, \alpha_{r}\right), 0\right)$ is a simply elliptic singularity, then the graph $\theta_{\alpha_{1}, \ldots, \alpha_{r}}$ is a Coxeter-Dynkin diagram of this singularity [E2]. Therefore we again obtain the identity $\phi_{M}(t)=p_{A}^{*}(t)$ (respectively $\left.\phi_{M}^{b}(t)=p_{A}^{*}(t)\right)$ in this case (cf. [E6]).

\section{§4. Fuchsian ICIS and Mirror Symmetry of K3 Surfaces}

We now consider Fuchsian ICIS. We first indicate the classification of Fuchsian ICIS.

Let $(X, x)$ be a Fuchsian singularity. If the algebra $A$ is generated by 3 elements, then $(X, x)$ is a hypersurface singularity in $\mathbb{C}^{3}$. These cases were classified by I. Dolgachev [D1], I. G. Sherbak [Sh], and Ph. Wagreich [Wag1]. If $A$ is generated by 4 elements, then one has an ICIS in $\mathbb{C}^{4}$. These cases were classified by $\mathrm{Ph}$. Wagreich [Wag2] (see also [Wag3]). In the case $g=0,(X, x)$ is a minimally elliptic singularity [Wag2, Proposition 5.5.1] and the classification can also be derived from H. Laufer's results [La]. There are a few more cases of ICIS of higher embedding dimension. More precisely we have:

Theorem 4. Let $(X, x)$ be a Fuchsian singularity with signature $\{g$; $\left.\alpha_{1}, \ldots, \alpha_{r}\right\}$. There is an ICIS with this signature if and only if the signature satisfies one of the following conditions: 
(i) $g=0, r=3, \alpha_{1}=2, \alpha_{2}=3$, and $7 \leq \alpha_{3} \leq 10$;

(ii) $g=0, r=3, \alpha_{1}=2, \alpha_{2} \geq 4$, and $9 \leq \alpha_{2}+\alpha_{3} \leq 12$;

(iii) $g=0, r=3, \alpha_{1}, \alpha_{2}, \alpha_{3} \geq 3$, and $10 \leq \alpha_{1}+\alpha_{2}+\alpha_{3} \leq 13$;

(iv) $g=0, r \geq 4$ and $9 \leq \alpha_{1}+\alpha_{2}+\cdots+\alpha_{r} \leq 12$;

(v) $g=1, r \geq 1, \alpha_{1}+\alpha_{2}+\cdots+\alpha_{r} \leq r+4$;

(vi) $2 \leq g \leq 4, r \geq 0, \alpha_{1}+\alpha_{2}+\cdots+\alpha_{r} \leq r+4-g$;

(vii) $g=5, r=0$.

Proof. The algebra $A$ is generated by 3 or 4 elements if and only if we have one of the cases (i)-(vi) and $Z$ is non-hyperelliptic in the cases $3 ; 2$ and $4 ;$. In the remaining cases one can easily show that there is an ICIS with these invariants, using [Wag2, Theorem 3.3]. Conversely, we show that in the other cases there are no ICIS. The embedding dimension is given in [Wag2, loc.cit.]. If $g=0$, then the singularity $(X, x)$ is minimally elliptic and the result follows from [La, Theorem 3.13]. In the remaining cases we can apply [VD, Lemma 3.9] to show that $(X, x)$ is not an ICIS if the signature does not satisfy the conditions of Theorem 4 . This proves Theorem 4 .

This leads to the following classification. The cases $g=0$ and $r \leq 4$ are listed in Table 1, the remaining cases in Table 2. Here we use the following notation. We first list the orbit invariants. For $g \geq 3$ we add (h) or (nh) to indicate whether $Z$ is hyperelliptic or non-hyperelliptic respectively. In the second column we give the weights and the degrees of the singularity. In the third column we indicate the name of the singularity according to Arnold's [A2] or Wall's notation [Wal1], [Wal2], if it exists. In column 4 we list the equation(s) of the singularity. Here $a$ is a complex number with $a \neq 0,1$. The cases $g=0$ and $r \leq 4$ are Kodaira singularities in the sense of [EW]. They were already considered in [E4]. The remaining cases with $g=0$ are still minimally elliptic and equations are given in [E1] and [Wal3] respectively. Finally we indicate the Milnor number $\mu$.

Remark 2. A Fuchsian singularity with signature $\{5 ;\}$ is the cone over a canonical curve of genus 5 in $\mathbb{P}^{4}$. As R.-O. Buchweitz pointed out to me, this is in general not an ICIS, but given by the Pfaffians of a $5 \times 5$ skew symmetric matrix.

Now let $(X, x)$ be a Fuchsian ICIS. All Fuchsian ICIS in $\mathbb{C}^{3}$ and $\mathbb{C}^{4}$ have equations such that one of the Theorems 1,2 , and 3 is applicable. The remaining singularities are 3 ICIS with hyperelliptic $Z$ and the singularity 5 ; (nh). There is the following relation between the singularities with the same signature, but with hyperelliptic $Z$ on the one hand and non-hyperelliptic $Z$ on the 
Table 1. Fuchsian ICIS with $g=0$ and $r \leq 4$.

\begin{tabular}{|c|c|c|c|c|}
\hline$g ; \alpha_{1}, \ldots, \alpha_{r}$ & Weights & Name & Equation(s) & $\mu$ \\
\hline $0 ; 2,3,7$ & $6,14,21 / 42$ & $E_{12}$ & $x^{7}+y^{3}+z^{2}$ & 12 \\
\hline $0 ; 2,3,8$ & $6,8,15 / 30$ & $Z_{11}$ & $x^{5}+x y^{3}+z^{2}$ & 11 \\
\hline $0 ; 2,3,9$ & $6,8,9 / 24$ & $Q_{10}$ & $x^{4}+x z^{2}+y^{3}$ & 10 \\
\hline $0 ; 2,3,10$ & $6,8,9,10 / 16,18$ & $J_{9}^{\prime}$ & $\left.\begin{array}{c}x w+y^{2} \\
x^{3}+y w+z^{2}\end{array}\right\}$ & 9 \\
\hline $0 ; 2,4,5$ & $4,10,15 / 30$ & $E_{13}$ & $x^{5} y+y^{3}+z^{2}$ & 13 \\
\hline $0 ; 2,4,6$ & $4,6,11 / 22$ & $Z_{12}$ & $x^{4} y+x y^{3}+z^{2}$ & 12 \\
\hline $0 ; 2,4,7$ & $4,6,7 / 18$ & $Q_{11}$ & $x^{3} y+x z^{2}+y^{3}$ & 11 \\
\hline $0 ; 2,4,8$ & $4,6,7,8 / 12,14$ & $J_{10}^{\prime}$ & $\begin{array}{c}x w+y^{2} \\
x^{2} y+y w+z^{2}\end{array}$ & 10 \\
\hline $0 ; 2,5,5$ & $4,5,10 / 20$ & $W_{12}$ & $x^{5}+y^{4}+z^{2}$ & 12 \\
\hline $0 ; 2,5,6$ & $4,5,6 / 16$ & $S_{11}$ & $x^{4}+x z^{2}+y^{2} z$ & 11 \\
\hline $0 ; 2,5,7$ & $4,5,6,7 / 11,12$ & $L_{10}$ & $\begin{array}{c}x w+y z \\
x^{3}+y w+z^{2}\end{array}$ & 10 \\
\hline $0 ; 2,6,6$ & $4,5,6,6 / 10,12$ & $K_{10}^{\prime}$ & $\begin{array}{c}x w+y^{2} \\
x^{3}+z^{2}+w^{2}\end{array}$ & 10 \\
\hline $0 ; 3,3,4$ & $3,8,12 / 24$ & $E_{14}$ & $x^{8}+y^{3}+z^{2}$ & 14 \\
\hline $0 ; 3,3,5$ & $3,5,9 / 18$ & $Z_{13}$ & $x^{6}+x y^{3}+z^{2}$ & 13 \\
\hline $0 ; 3,3,6$ & $3,5,6 / 15$ & $Q_{12}$ & $x^{5}+x z^{2}+y^{3}$ & 12 \\
\hline $0 ; 3,3,7$ & $3,5,6,7 / 10,12$ & $J_{11}^{\prime}$ & $\left.\begin{array}{c}x w+y^{2} \\
x^{4}+y w+z^{2}\end{array}\right\}$ & 11 \\
\hline $0 ; 3,4,4$ & $3,4,8 / 16$ & $W_{13}$ & $x^{4} y+y^{4}+z^{2}$ & 13 \\
\hline $0 ; 3,4,5$ & $3,4,5 / 13$ & $S_{12}$ & $x^{3} y+x z^{2}+y^{2} z$ & 12 \\
\hline $0 ; 3,4,6$ & $3,4,5,6 / 9,10$ & $L_{11}$ & $\begin{array}{c}x w+y z \\
x^{2} y+y w+z^{2}\end{array}$ & 11 \\
\hline $0 ; 3,5,5$ & $3,4,5,5 / 8,10$ & $K_{11}^{\prime}$ & $\left\{\begin{array}{c}x w+y^{2} \\
x^{2} y+z^{2}+w^{2}\end{array}\right.$ & 11 \\
\hline $0 ; 4,4,4$ & $3,4,4 / 12$ & $U_{12}$ & $x^{4}+y^{3}+z^{3}$ & 12 \\
\hline $0 ; 4,4,5$ & $3,4,4,5 / 8,9$ & $M_{11}$ & $\left\{\begin{array}{c}x w+y z \\
x^{3}+(y+z) w\end{array}\right.$ & 11 \\
\hline
\end{tabular}

(continued) 
(continued)

\begin{tabular}{|c|c|c|c|c|}
\hline $0 ; 2,2,2,3$ & $2,6,9 / 18$ & $J_{3,0}$ & $x^{9}+y^{3}+z^{2}$ & 16 \\
\hline $0 ; 2,2,2,4$ & $2,4,7 / 14$ & $Z_{1,0}$ & $x^{7}+x y^{3}+z^{2}$ & 15 \\
\hline $0 ; 2,2,2,5$ & $2,4,5 / 12$ & $Q_{2,0}$ & $x^{6}+x z^{2}+y^{3}$ & 14 \\
\hline $0 ; 2,2,2,6$ & $2,4,5,6 / 8,10$ & $J_{2,0}^{\prime}$ & $\left.\begin{array}{c}x w+y^{2} \\
x^{5}+x y^{2}+y w+z^{2}\end{array}\right\}$ & 13 \\
\hline $0 ; 2,2,3,3$ & $2,3,6 / 12$ & $W_{1,0}$ & $x^{6}+y^{4}+z^{2}$ & 15 \\
\hline $0 ; 2,2,3,4$ & $2,3,4 / 10$ & $S_{1,0}$ & $x^{5}+x z^{2}+y^{2} z$ & 14 \\
\hline $0 ; 2,2,3,5$ & $2,3,4,5 / 7,8$ & $L_{1,0}$ & $\left.\begin{array}{c}x w+y z \\
x^{4}+x y^{2}+y w+z^{2}\end{array}\right\}$ & 13 \\
\hline $0 ; 2,2,4,4$ & $2,3,4,4 / 6,8$ & $K_{1,0}^{\prime}$ & $\left.\begin{array}{c}x w+y^{2} \\
x^{4}+x y^{2}+z^{2}+w^{2}\end{array}\right\}$ & 13 \\
\hline $0 ; 2,3,3,3$ & $2,3,3 / 9$ & $U_{1,0}$ & $x^{3} y+y^{3}+z^{3}$ & 14 \\
\hline $0 ; 2,3,3,4$ & $2,3,3,4 / 6,7$ & $M_{1,0}$ & $\begin{array}{c}x w+y z \\
\left(x^{2}+w\right)(y+z)\end{array}$ & 13 \\
\hline $0 ; 3,3,3,3$ & $2,3,3,3 / 6,6$ & $I_{1,0}$ & $\begin{array}{c}x^{3}+(y-z) w \\
a x^{3}+y(z-w)\end{array}$ & 13 \\
\hline
\end{tabular}

other hand. If the one with non-hyperelliptic $Z$ has weights $q_{1}, \ldots, q_{n}$ and degrees $d_{1}, \ldots, d_{n-2}$, then the one with the same signature but hyperelliptic $Z$ has weights $q_{1}, \ldots, q_{n}, q_{1}^{\prime}, \ldots, q_{m}^{\prime}$ and degrees $d_{1}, \ldots, d_{n-2}, q_{1}^{\prime}, \ldots, q_{m}^{\prime}$ for some $m$. But this means that both singularities have the same Poincaré series and the same characteristic polynomial of the monodromy and Theorem 2 or Theorem 3 is also applicable in this case. Finally, for the singularity $5 ;(\mathrm{nh})$ we have $\phi_{M}(t)=\left(1-t^{2}\right)^{16} /(1-t)$ and we define $\phi_{M}^{b}(t):=\phi_{M}(t) /(1-t)^{7}=$ $\left(1-t^{2}\right)^{16} /(1-t)^{8}$ and $\tilde{\phi}_{A}(t):=\left(1-t^{2}\right)^{8} /(1-t)^{16}$. If $(X, x)$ is a hypersurface singularity, then we set $\phi_{M}^{b}(t):=\phi_{M}(t)$. Therefore for all Fuchsian ICIS, polynomials $\tilde{\phi}_{A}(t)$ and $\phi_{M}^{b}(t)$ are defined and we obtain:

Corollary 1. If $(X, x)$ is a Fuchsian ICIS, then one has

$$
\tilde{\phi}_{A}^{*}(t)=\phi_{M}^{b}(t)
$$

Let $(X, x)$ be one of the 14 exceptional unimodal hypersurface singularities. Then $\tilde{\phi}_{A}(t)=\phi_{A}(t)$ and $\phi_{M}^{b}(t)=\phi_{M}(t)$. According to Proposition 1 , $\phi_{A}(t)$ is the characteristic polynomial of the monodromy operator of the dual singularity in Arnold's strange duality and the relation of Corollary 1 was already observed by K. Saito [Sa1], [Sa2]. In the case $g=0$ and $r \leq 4$ but $(X, x)$ 
Table 2. Fuchsian ICIS with $g=0$ and $r \geq 5$ or $g>0$.

\begin{tabular}{|c|c|c|c|c|}
\hline$g ; \alpha_{1}, \ldots, \alpha_{r}$ & Weights & Name & Equation(s) & $\mu$ \\
\hline $0 ; 2,2,2,2,2$ & $2,2,5 / 10$ & $N A_{0,0}^{1}$ & $x^{5}+y^{5}+z^{2}$ & 16 \\
\hline $0 ; 2,2,2,2,3$ & $2,2,3 / 8$ & $V N A_{0,0}^{1}$ & $x^{4}+y^{4}+y z^{2}$ & 15 \\
\hline $0 ; 2,2,2,2,4$ & $2,2,3,4 / 6,6$ & $\alpha 1^{(1)}$ & $\begin{array}{c}x+y w \\
x w+y^{3}+z^{2}\end{array}$ & 14 \\
\hline $0 ; 2,2,2,3,3$ & $2,2,3,3 / 5,6$ & $\alpha 1^{(2)}$ & $x w+y z$ & 14 \\
\hline $0 ; 2,2,2,2,2,2$ & $2,2,2,3 / 4,6$ & $\delta 1$ & $\left.\begin{array}{l}x y+z^{2} \\
y^{3}+z^{3}+y^{2}\end{array}\right\}$ & 15 \\
\hline $1 ; 2$ & $1,4,6 / 12$ & $J_{4,0}$ & $x^{12}+y^{3}+z^{2}$ & 22 \\
\hline $1 ; 3$ & $1,3,5 / 10$ & $Z_{2,0}$ & $x^{10}+x y^{3}+z^{2}$ & 21 \\
\hline $1 ; 4$ & $1,3,4 / 9$ & $Q_{3,0}$ & $x^{9}+x z^{2}+y^{3}$ & 20 \\
\hline $1 ; 5$ & $1,3,4,5 / 6,8$ & $J_{3,0}^{\prime}$ & $\begin{array}{l}x w+y^{2} \\
8+y w+z^{2}\end{array}$ & 19 \\
\hline $1 ; 2,2$ & $1,2,4 / 8$ & $X_{2,0}$ & $x^{8}+y^{4}+z^{2}$ & 21 \\
\hline $1 ; 2,3$ & $1,2,3 / 7$ & $S_{2,0}^{*}$ & $x^{7}+x z^{2}+y^{2} z$ & 20 \\
\hline $1 ; 2,4$ & $1,2,3,4 / 5,6$ & $L_{2,0}^{*}$ & $\begin{array}{c}x w+y z \\
x^{6}+u y+z^{2}\end{array}$ & 19 \\
\hline $1 ; 3,3$ & $1,2,3,3 / 4,6$ & $K^{\prime} X_{2,0}$ & $\begin{array}{c}x w+y^{2} \\
x^{6}\end{array}$ & 19 \\
\hline $1 ; 2,2,2$ & $1,2,2 / 6$ & $U_{2,0}^{*}$ & $x^{6}+y^{3}+z^{3}$ & 20 \\
\hline $1 ; 2,2,3$ & $1,2,2,3 / 4,5$ & $M_{2,0}^{*}$ & $\begin{array}{c}x w+y z \\
\left(x^{3}\right.\end{array}$ & 19 \\
\hline $1 ; 2,2,2,2$ & $1,2,2,2 / 4,4$ & $I T_{2,2,2,2}$ & $\begin{array}{c}x^{4}+(y-z) w \\
x^{4}+u(z-w)\end{array}$ & 19 \\
\hline 2 ; & $1,1,3 / 6$ & & $x^{6}+y^{6}+z^{2}$ & 25 \\
\hline $2 ; 2$ & $1,1,2 / 5$ & & $x^{5}+x z^{2}+y^{5}$ & 24 \\
\hline $2 ; 3$ & $1,1,2,3 / 4,4$ & & $\begin{array}{c}x w+y^{4} \\
x^{4}+y w+z^{2}\end{array}$ & 23 \\
\hline $2 ; 2,2$ & $1,1,2,2 / 3,4$ & & $\left.\begin{array}{c}x w+y z \\
x^{4}+u^{4}\end{array}\right\}$ & 23 \\
\hline $3 ;(\mathrm{nh})$ & $1,1,1 / 4$ & & $x^{4}+y^{4}+z^{4}$ & 27 \\
\hline $3 ;(\mathrm{h})$ & $1,1,1,2 / 2,4$ & & $\begin{array}{c}x y+z^{2} \\
4\end{array}$ & 27 \\
\hline $3 ; 2(\mathrm{nh})$ & $1,1,1,2 / 3,3$ & & $\left\{\begin{array}{c}x w+y^{3} \\
x^{3}\end{array}\right\}$ & 26 \\
\hline $3 ; 2(\mathrm{~h})$ & $1,1,1,2,2 / 2,3,3$ & & & 26 \\
\hline $4 ;(\mathrm{nh})$ & $1,1,1,1 / 2,3$ & & $\left.\begin{array}{c}x w+y z \\
x^{3}\end{array}\right\}$ & 29 \\
\hline $4 ;(\mathrm{h})$ & $\begin{array}{c}1,1,1,1,2,2 / \\
2,2,2,3\end{array}$ & & & 29 \\
\hline $5 ;(\mathrm{nh})$ & $\begin{array}{c}1,1,1,1,1 / \\
2,2,2\end{array}$ & & 3 quadrics in $\mathbb{C}^{5}$ & 31 \\
\hline $5 ;(\mathrm{h})$ & $\begin{array}{c}1,1,1,1,1,2,2,2 / \\
2,2,2,2,2,2\end{array}$ & & & 31 \\
\hline
\end{tabular}


is not a unimodal hypersurface singularity, we have the extension of Arnold's strange duality considered in [EW]. In this case, we have again $\tilde{\phi}_{A}(t)=\phi_{A}(t)$. From Proposition 1 and [E4] we can derive the following results. If $(X, x)$ is a hypersurface singularity with $r=4$, then $\phi_{A}(t)$ is equal to the polynomial $\phi_{M}^{b}(t)$ of the dual ICIS. If $(X, x)$ is an ICIS with $r=3$, then $\phi_{A}(t)$ is equal to the characteristic polynomial of the monodromy operator of the dual "virtual" singularity considered in $[\mathrm{EW}]$. Finally, if $(X, x)$ is an ICIS with $r=4$, then $\phi_{A}(t)$ is equal to the polynomial $\phi_{M}^{b}(t)$ of the dual "virtual" ICIS.

There is the following relation of the duality of Corollary 1 with the mirror symmetry of $K 3$ surfaces.

Let $(X, x)$ be a normal surface singularity with good $\mathbb{C}^{*}$-action. According to $[\mathrm{P} 1]$, the $\mathbb{C}^{*}$-action on $X$ extends (at least formally) to a $\mathbb{C}^{*}$-action on a semiuniversal deformation $p: \mathcal{X} \rightarrow \mathcal{S}$ of $(X, x)$. Assume that $(X, x)$ is smoothable. Then also the Milnor fibre $\mathcal{X}_{t}$ can be compactified in a natural way to a surface $\overline{\mathcal{X}}_{t}$ with the same cyclic quotient singularities as $\bar{X}$ along a curve at infinity isomorphic to $\bar{X}_{\infty}$. Denote by $Y$ the minimal resolution of $\overline{\mathcal{X}}_{t}$. By $[\mathrm{P} 4,6.13$ Theorem], $Y$ is a minimal $K 3$ surface if and only if $(X, x)$ is Fuchsian.

Let $(X, x)$ be a Fuchsian ICIS. Let $Y^{-}$be a tubular neighbourhood of the curve at infinity $Y_{\infty} \cong \tilde{X}_{\infty}$, and set $Y^{+}:=Y \backslash \operatorname{int}\left(Y^{-}\right)$and $\Sigma:=\partial Y^{+}=$ $\partial Y^{-}=Y^{+} \cap Y^{-}$. Then the Mayer-Vietoris sequence of the pair $\left(Y^{+}, Y^{-}\right)$gives the following exact sequence (we consider homology with integral coefficients):

$0 \rightarrow H_{2}(\Sigma) \rightarrow H_{2}\left(Y^{+}\right) \oplus H_{2}\left(Y^{-}\right) \rightarrow H_{2}(Y) \rightarrow H_{1}(\Sigma) \rightarrow H_{1}\left(Y^{+}\right) \oplus H_{1}\left(Y^{-}\right) \rightarrow 0$.

Now $M:=H_{2}\left(Y^{+}\right)$is the Milnor lattice. The group $H_{2}(\Sigma)$ is the radical $M_{0}:=\operatorname{rad}(M)$ of $M$ (cf. e.g. [HM]). The rank of $M_{0}$ is $\mu_{0}=2 g$. Let $H_{1}(\Sigma)_{\text {tors }}$ denote the torsion subgroup of $H_{1}(\Sigma)$. Then $H_{1}(\Sigma) / H_{1}(\Sigma)_{\text {tors }} \cong H_{1}\left(Y^{-}\right) \cong$ $\mathbb{Z}^{\mu_{0}}$ and $H_{1}\left(Y^{+}\right)=0$. This shows that we have a primitive embedding of the non-degenerate lattice $M / M_{0}$ corresponding to the Milnor lattice into the homology lattice $K:=H_{2}(Y) \cong\left(-E_{8}\right) \oplus\left(-E_{8}\right) \oplus U \oplus U \oplus U$ of a $K 3$ surface (cf. [P3]). By a result of V. V. Nikulin [N], this embedding is unique up to isometries. Moreover, the orthogonal complement of $M / M_{0}$ in $K$ is the lattice $H_{2}\left(Y^{-}\right)=M_{\infty}$.

The duality between the lattices $M / M_{0}$ and $M_{\infty}$ corresponds to the mirror symmetry of $K 3$-surfaces, see [D5]. In the case when $g=0$ and $r \leq 4$, the lattice $M_{\infty} \oplus U$ is related to the Milnor lattice and $c_{\infty}$ to the monodromy operator of another Fuchsian singularity $\left(X^{*}, x^{*}\right)$ with $g=0$ and $r \leq 4$ and we obtain Arnold's strange duality and its extension [E4]. It is not known to the author whether $M_{\infty} \oplus U$ and $c_{\infty}$ correspond to a singularity in the other cases. 


\section{$\S 5 . \quad$ Singular Moonshine}

We shall now discuss relations to the Leech lattice.

For a polynomial

$$
\phi(t)=\prod_{m \mid h}\left(1-t^{m}\right)^{\chi_{m}}
$$

where $\chi_{m} \in \mathbb{Z}$, we use the symbolic notation

$$
\pi:=\prod_{m \mid h} m^{\chi_{m}} .
$$

In the theory of finite groups, this symbol is known as a Frame shape (cf. [CN]).

Let $(X, x)$ be a singularity which satisfies the assumptions of Theorem 1 , 2, or 3. Let $\pi_{M}$ and $\pi_{M}^{b}$ denote the Frame shapes corresponding to the polynomials $\phi_{M}(t)$ and $\phi_{M}^{b}(t)$ respectively. If $(X, x)$ is a hypersurface singularity, then we put $\pi:=\pi_{M}$. Otherwise we define $\pi:=\pi_{M}^{b}$ according to Theorem 2 or 3 .

Now let $(X, x)$ be a Fuchsian ICIS. All Fuchsian ICIS in $\mathbb{C}^{3}$ and $\mathbb{C}^{4}$ have equations such that one of the Theorems 1, 2, and 3 is applicable. Above we also defined a polynomial $\phi_{M}^{b}$ in the remaining cases and we set $\pi:=\pi_{M}^{b}$ in these cases as well. Then we make the following observation.

Theorem 5. Let $(X, x)$ be a Fuchsian ICIS. Then the symbol $\pi \pi^{*}$ is a 24-dimensional self-dual Frame shape which is the Frame shape of an automorphism of the Leech lattice.

If $(X, x)$ is one of the 14 exceptional hypersurface singularities, then $\pi=$ $\pi_{M}$ and it was already observed by K. Saito in [Sa1], [Sa2] that $\pi \pi^{*}$ is the Frame shape of an automorphism of the Leech lattice. If $(X, x)$ is a Kodaira singularity (i.e., $g=0$ and $r \leq 4$ ), then the corresponding result can already be found in [E4].

In [Sa2, Appendix 1] Saito considers regular systems of weights of rank 24 having negative exponents. They correspond to quasihomogeneous hypersurface singularities in $\mathbb{C}^{3}$ with $R \geq 1$ and $\mu=24$. He finds 11 cases with $R>1$. It can be checked using the normal forms of quasihomogeneous functions in three variables $[\mathrm{A} 1,13.2]$ that the list is complete up to one case which was omitted. The complete list is in Table 3. By a computer search we found 13 quasihomogeneous ICIS in $\mathbb{C}^{4}$ with $\mu=25$. They are listed in Table 4 . We use the same conventions as for Tables 1 and 2. All singularities have $\mu_{0}=g=0$, $p_{g}=2$, and hence $\mu_{+}=4$. 
Table 3. Quasihomogeneous ICIS in $\mathbb{C}^{3}$ with $\mu=24$.

\begin{tabular}{|c|c|c|c|c|c|c|}
\hline$g ; \alpha_{1}, \ldots, \alpha_{r}$ & $b$ & $R$ & Weights & Name & Equation & \\
\hline $0 ; 7,7,7$ & 1 & 4 & $3,7,7 / 21$ & $U_{24}$ & $x^{7}+y^{3}+z^{3}$ & $(\mathrm{~b}, \mathrm{e})$ \\
$0 ; 3,7,11$ & 1 & 4 & $3,7,11 / 25$ & $S_{24}$ & $x^{6} y+x z^{2}+y^{2} z$ & $(\mathrm{~b})$ \\
$0 ; 3,3,15$ & 1 & 4 & $3,11,15 / 33$ & $Q_{24}$ & $x^{11}+x z^{2}+y^{3}$ & $(\mathrm{~b}, \mathrm{e})$ \\
$0 ; 4,7,9$ & 1 & 5 & $4,7,9 / 25$ & $V^{\sharp} N C_{18}^{1}$ & $x^{4} z+x y^{3}+y z^{2}$ & $(\mathrm{~b})$ \\
$0 ; 4,4,12$ & 1 & 5 & $4,7,12 / 28$ & $V N C_{18}^{1}$ & $x^{7}+x z^{2}+y^{4}$ & $(\mathrm{~b}, \mathrm{e})$ \\
$0 ; 2,9,9$ & 1 & 5 & $4,9,18 / 36$ & $W_{24}$ & $x^{9}+y^{4}+z^{2}$ & $(\mathrm{~b}, \mathrm{e})$ \\
$0 ; 2,4,14$ & 1 & 5 & $4,14,23 / 46$ & $Z_{24}$ & $x^{8} y+x y^{3}+z^{2}$ & $(\mathrm{~b}, \mathrm{e})$ \\
$0 ; 3,6,9$ & 1 & 7 & $6,9,11 / 33$ & $V^{\prime}\left(Z_{12}\right)^{2}$ & $x^{4} y+x y^{3}+z^{3}$ & $(\mathrm{~b}, \mathrm{e})$ \\
$0 ; 2,6,10$ & 1 & 7 & $6,10,23 / 46$ & $N C_{17}^{1}$ & $x^{6} y+x y^{4}+z^{2}$ & $(\mathrm{~b}, \mathrm{e})$ \\
$0 ; 2,3,13$ & 1 & 7 & $6,26,39 / 78$ & $E_{24}$ & $x^{13}+y^{3}+z^{2}$ & $(\mathrm{~b}, \mathrm{e})$ \\
$0 ; 2,5,7$ & 1 & 11 & $10,14,35 / 70$ & $N F_{(4)}^{1}$ & $x^{7}+y^{5}+z^{2}$ & $(\mathrm{~b}, \mathrm{e})$ \\
$0 ; 3,4,5$ & 1 & 13 & $12,15,20 / 60$ & $V^{\prime}\left(W_{12}\right)^{2}$ & $x^{5}+y^{4}+z^{3}$ & $(\mathrm{~b}, \mathrm{e})$ \\
\hline
\end{tabular}

Saito already observed that the Frame shapes $\pi$ of the hypersurface singularities are self-dual and appear as Frame shapes of automorphisms of the Leech lattice. For 8 of the ICIS in $\mathbb{C}^{4}$ with $\mu=25$ we can apply Theorem 2 or 3 and define $\pi:=\pi_{M}^{b}$. This symbol is also a self-dual Frame shape of dimension 24 which appears as the Frame shape of an automorphism of the Leech lattice. In the remaining cases either Theorems 2 and 3 are not applicable and so $\pi_{M}^{b}$ is not defined or $\pi_{M}^{b}$ is not self-dual.

In Table 5 we have listed the 39 self-dual Frame shapes of the automorphism group $\cdot 0$ of the Leech lattice. We use the ATLAS notation [ATL] for the conjugacy classes. We consider the following five constructions. For a Frame shape $\pi=\prod_{m \mid h} m^{\chi_{m}}$ we call the minimal $h$ the order of $\pi$ and $\operatorname{deg} \pi:=\sum_{m \mid h} m \chi_{m}$ the degree of $\pi$.

(a) Consider any combination (direct sum) $\pi=\pi_{1} \cdots \pi_{s}$ of the (self-dual) Frame shapes of the Coxeter elements of the root systems of type $A_{l}, D_{l}, E_{6}$, $E_{7}$, or $E_{8}$ such that the orders of the $\pi_{i}$ are the same and the degree of $\pi$ is equal to 24 . (There are 23 such combinations; they correspond to the 23 Niemeier lattices, see e.g. [E3, Proposition 3.4].)

(b) Consider the (self-dual) symbol $\pi$ of a singularity of Table 3 or 4 respectively for which (b) is indicated in the last column.

(c) Consider the symbol $\pi \pi^{*}$ where $\pi=\pi_{1} \cdots \pi_{s}$ is any combination (direct sum) of Frame shapes of the simply elliptic singularities (cf. [E6]) such that the orders of the $\pi_{i}$ are the same and the degree of $\pi$ is equal to 24. (There are 
Table 4. Quasihomogeneous ICIS in $\mathbb{C}^{4}$ with $\mu=25$.

\begin{tabular}{|c|c|c|c|c|c|c|}
\hline$g ; \alpha_{i}$ & $b$ & $R$ & Weights & Name & Equations & \\
\hline $0 ; 5,5,5,5$ & 2 & 3 & $2,5,5,5 / 10,10$ & $I_{1,0}^{2}$ & $\left\{\begin{array}{c}x^{5}+(y-z) w \\
a x^{5}+y(z-w)\end{array}\right.$ & $(\mathrm{b}, \mathrm{e})$ \\
\hline $0 ; 2,5,5,8$ & 2 & 3 & $2,5,5,8 / 10,13$ & $M_{2,0}$ & $\begin{array}{c}x w+y z \\
\left(x^{4}+w\right)(y+z)\end{array}$ & (b) \\
\hline $0 ; 2,2,8,8$ & 2 & 3 & $2,5,8,8 / 10,16$ & $K^{\prime} W_{2,0}$ & $\begin{array}{c}x z+y^{2} \\
x^{8}+z^{2}+w^{2}\end{array}$ & $(\mathrm{~b}, \mathrm{e})$ \\
\hline $0 ; 2,2,5,11$ & 2 & 3 & $2,5,8,11 / 13,16$ & $L_{2,0}$ & $\begin{array}{c}x w+y z \\
x^{8}+y w+z^{2}\end{array}$ & (b) \\
\hline $0 ; 2,2,2,14$ & 2 & 3 & $2,8,11,14 / 16,22$ & $J_{4,0}^{\prime}$ & $\left\{\begin{array}{c}x w+y^{2} \\
x^{11}+y w+z^{2}\end{array}\right.$ & $(\mathrm{b}, \mathrm{e})$ \\
\hline $0 ; 3,3,5,7$ & 2 & 4 & $3,5,6,7 / 12,13$ & & $\begin{array}{c}y w+z^{2} \\
x^{2} w+x y^{2}+z w\end{array}$ & \\
\hline $0 ; 3,3,3,9$ & 2 & 4 & $3,5,6,9 / 12,15$ & & $\begin{array}{c}x w+z^{2} \\
x^{5}+y^{3}+z w\end{array}$ & (e) \\
\hline $0 ; 2,4,4,6$ & 2 & 5 & $4,6,7,8 / 14,16$ & & $\begin{array}{c}y w+z^{2} \\
x^{4}+x y^{2}+w^{2}\end{array}$ & (e) \\
\hline $0 ; 2,3,4,7$ & 2 & 5 & $4,6,7,9 / 13,18$ & & $\begin{array}{c}x w+y z \\
x z^{2}+y^{3}+w^{2}\end{array}$ & (b) \\
\hline $0 ; 2,2,4,8$ & 2 & 5 & $4,6,8,11 / 12,22$ & & $\left\{\begin{array}{c}x^{3}+y^{2}+x z \\
x y^{3}+x^{4} y+y z^{2}+w^{2}\end{array}\right\}$ & (b) \\
\hline $0 ; 2,3,3,4$ & 2 & 7 & $6,8,9,12 / 18,24$ & & $\left\{\begin{array}{c}x w+z^{2} \\
x^{4}+x z^{2}+y^{3}+w^{2}\end{array}\right.$ & \\
\hline $0 ; 2,2,3,5$ & 2 & 7 & $6,8,10,15 / 16,30$ & & $\left\{\begin{array}{c}x z+y^{2} \\
x^{5}+x y^{3}+z^{3}+w^{2}\end{array}\right\}$ & $(\mathrm{b}, \mathrm{e})$ \\
\hline $0 ; 3,4,20$ & 1 & 11 & $9,12,16,20 / 32,36$ & & $\left\{\begin{array}{c}y w+z^{2} \\
x^{4}+y^{3}+z w\end{array}\right.$ & \\
\hline
\end{tabular}


Table 5. Self-dual Frame shapes of $\cdot 0$.

\begin{tabular}{|c|c|c|}
\hline$\overline{\text { ATL }}$ & Frame shape & realized \\
\hline $1 \mathrm{~A}$ & $2^{24} / 1^{24}$ & (a) $A_{1}^{24}$ (c) $\tilde{D}_{5}^{4}$ (d) $\{5 ;\}$ \\
\hline $3 \mathrm{~A}$ & $3^{12} / 1^{12}$ & (a) $A_{2}^{12}$ (c) $\tilde{E}_{6}^{3}$ (d) $\{3 ; 2\},\{4 ;\}$ \\
\hline $3 \mathrm{~B}$ & $2^{6} 6^{6} / 1^{6} 3^{6}$ & (a) $D_{4}^{6}$ (d) $\delta 1$ \\
\hline $4 \mathrm{~A}$ & $4^{8} / 1^{8}$ & (a) $A_{3}^{8}$ (d) $I T_{2,2,2,2},\{2 ; 3\},\{2 ; 22\},\{3 ;\}$ \\
\hline $5 \mathrm{~A}$ & $5^{6} / 1^{6}$ & (a) $A_{4}^{6}$ (d) $M_{2,0}^{*},\{2 ; 2\}$ (e) $I_{1,0}^{2}$ \\
\hline $5 \mathrm{~B}$ & $2^{4} 10^{4} / 1^{4} 5^{4}$ & (a) $D_{6}^{4}$ (d) $J_{2,0}^{\prime}, N A_{0,0}^{1}$ \\
\hline $6 \mathrm{~A}$ & $3^{4} 6^{4} / 1^{4} 2^{4}$ & (d) $I_{1,0}$ \\
\hline $6 \mathrm{D}$ & $2 \cdot 6^{5} / 1^{5} 3$ & $\begin{array}{l}\text { (a) } A_{5}^{4} D_{4} \\
\text { (d) } \alpha 1^{(1)}, \alpha 1^{(2)}, L_{2,0}^{*}, K^{\prime} X_{2,0}, U_{2,0}^{*},\{2 ;\}\end{array}$ \\
\hline $7 \mathrm{~A}$ & $7^{4} / 1^{4}$ & (a) $A_{6}^{4}$ (d) $M_{1,0}, S_{2,0}^{*}$ (e) $U_{24}$ \\
\hline $7 \mathrm{~B}$ & $2^{3} 14^{3} / 1^{3} 7^{3}$ & (a) $D_{8}^{3}$ (d) $J_{10}^{\prime}, Z_{1,0}$ (e) $J_{4,0}^{\prime}$ \\
\hline $8 \mathrm{C}$ & $2^{2} 8^{4} / 1^{4} 4^{2}$ & $\begin{array}{l}\text { (a) } A_{7}^{2} D_{5}^{2} \text { (d) } L_{1,0}, K_{1,0}^{\prime}, V N A_{0,0}^{1}, J_{3,0}^{\prime}, X_{2,0} \\
\text { (e) } K^{\prime} W_{2,0}\end{array}$ \\
\hline $9 \mathrm{~A}$ & $9^{3} / 1^{3}$ & (a) $A_{8}^{3}$ (d) $M_{11}, U_{1,0}, Q_{3,0}$ (e) $\{0 ; 3339\}$ \\
\hline $9 \mathrm{C}$ & $2^{3} 3^{2} 18^{3} / 1^{3} 6^{2} 9^{3}$ & (a) $D_{10} E_{7}^{2}$ (d) $J_{9}^{\prime}, J_{3,0}$ \\
\hline $10 \mathrm{~A}$ & $5^{2} 10^{2} / 1^{2} 2^{2}$ & (b) $I_{1,0}^{2}$ \\
\hline $10 \mathrm{E}$ & $2 \cdot 10^{3} / 1^{3} 5$ & (a) $A_{9}^{2} D_{6}$ (d) $L_{11}, K_{11}^{\prime}, S_{1,0}, Z_{2,0}$ \\
\hline $11 \mathrm{~A}$ & $2^{2} 22^{2} / 1^{2} 11^{2}$ & (a) $D_{12}^{2}$ (b) $J_{4,0}^{\prime},\{0 ; 2248\}$ (d) $Z_{12}$ \\
\hline $12 \mathrm{~A}$ & $2^{4} 3^{4} 12^{4} / 1^{4} 4^{4} 6^{4}$ & (a) $E_{6}^{4}$ \\
\hline $12 \mathrm{E}$ & $4^{2} 12^{2} / 1^{2} 3^{2}$ & (d) $U_{12}$ (e) $V N C_{18}^{1},\{0 ; 2446\}$ \\
\hline $12 \mathrm{~K}$ & $2^{2} 3 \cdot 12^{3} / 1^{3} 4 \cdot 6^{2}$ & (a) $A_{11} D_{7} E_{6}$ \\
\hline & & (d) $L_{10}, K_{10}^{\prime}, J_{11}^{\prime}, Q_{2,0}, W_{1,0}, J_{4,0}$ \\
\hline $13 \mathrm{~A}$ & $13^{2} / 1^{2}$ & (a) $A_{12}^{2}$ (b) $M_{2,0}$ (d) $S_{12}$ \\
\hline $15 \mathrm{~A}$ & $2^{3} 3^{3} 5^{3} 30^{3} / 1^{3} 6^{3} 10^{3} 15^{3}$ & (a) $E_{8}^{3}$ \\
\hline $15 \mathrm{~B}$ & $3^{2} 15^{2} / 1^{2} 5^{2}$ & (d) $Q_{12}$ (e) $Q_{24}$ \\
\hline $15 \mathrm{D}$ & $2 \cdot 6 \cdot 10 \cdot 30 / 1 \cdot 3 \cdot 5 \cdot 15$ & (e) $N C_{17}^{1}$ \\
\hline $15 \mathrm{E}$ & $2^{2} 3 \cdot 5 \cdot 30^{2} / 1^{2} 6 \cdot 10 \cdot 15^{2}$ & (a) $D_{16} E_{8}(\mathrm{~b}, \mathrm{e})\{0 ; 2235\}$, (d) $Z_{11}, E_{13}$ \\
\hline $16 \mathrm{~B}$ & $2 \cdot 16^{2} / 1^{2} 8$ & (a) $A_{15} D_{9}$ (b) $K^{\prime} W_{2,0}, L_{2,0}$ (d) $S_{11}, W_{13}$ \\
\hline $18 \mathrm{~A}$ & $9 \cdot 18 / 1 \cdot 2$ & (e) $W_{24}, V^{\prime}\left(Z_{12}\right)^{2}$ \\
\hline $18 \mathrm{~B}$ & $2 \cdot 3 \cdot 18^{2} / 1^{2} 6 \cdot 9$ & (a) $A_{17} E_{7}$ (b) $\{0 ; 2347\}$ (d) $Q_{11}, Z_{13}$ \\
\hline $20 \mathrm{~A}$ & $2^{2} 5^{2} 20^{2} / 1^{2} 4^{2} 10^{2}$ & (d) $W_{12}$ \\
\hline $21 \mathrm{~A}$ & $2^{2} 3^{2} 7^{2} 42^{2} / 1^{2} 6^{2} 14^{2} 21^{2}$ & (d) $E_{12}$ \\
\hline
\end{tabular}

(continued) 
(continued)

\begin{tabular}{|l|c|l|}
\hline $21 \mathrm{~B}$ & $7 \cdot 21 / 1 \cdot 3$ & (b) $U_{24}$ \\
$23 \mathrm{~A}$ & $2 \cdot 46 / 1 \cdot 23$ & (a) $D_{24}(\mathrm{~b}) Z_{24}, N C_{17}^{1}$ \\
$24 \mathrm{~B}$ & $2 \cdot 3^{2} 4 \cdot 24^{2} / 1^{2} 6 \cdot 8^{2} 12$ & (d) $Q_{10}, E_{14}$ \\
$25 \mathrm{~A}$ & $25 / 1$ & (a) $A_{24}(\mathrm{~b}) S_{24}, V^{\sharp} N C_{18}^{1}$ \\
$28 \mathrm{~A}$ & $4 \cdot 28 / 1 \cdot 7$ & (b) $V N C_{18}^{1}(\mathrm{e}) Z_{24}$ \\
$33 \mathrm{~A}$ & $3 \cdot 33 / 1 \cdot 11$ & (b) $Q_{24}, V^{\prime}\left(Z_{12}\right)^{2}$ \\
$35 \mathrm{~A}$ & $2 \cdot 5 \cdot 7 \cdot 70 / 1 \cdot 10 \cdot 14 \cdot 35$ & (b,e) $N F_{(4)}^{1}$ \\
$36 \mathrm{~A}$ & $2 \cdot 9 \cdot 36 / 1 \cdot 4 \cdot 18$ & (b) $W_{24}$ \\
$39 \mathrm{~A}$ & $2 \cdot 3 \cdot 13 \cdot 78 / 1 \cdot 6 \cdot 26 \cdot 39$ & (b,e) $E_{24}$ \\
$60 \mathrm{~A}$ & $3 \cdot 4 \cdot 5 \cdot 60 / 1 \cdot 12 \cdot 15 \cdot 20$ & (b,e) $V^{\prime}\left(W_{12}\right)^{2}$ \\
& & \\
\hline
\end{tabular}

only two such combinations, namely $\tilde{D}_{5}^{4}$ and $\tilde{E}_{6}^{3}$.)

(d) Consider the symbol $\pi \pi^{*}$ where $\pi$ is the symbol of a Fuchsian ICIS according to Theorem 5 .

(e) Consider the symbol $\pi \pi^{*}$ where $\pi$ is the symbol corresponding to the polynomial $\psi_{A}(t)$ of a singularity of Table 3 or 4 for which (e) is indicated in the last column (cf. Remark 1).

By these constructions we get all of the self-dual Frame shapes of $\cdot 0$ (cf. also [Sa2, Appendix 1], where 4 cases do not appear). The different realizations are indicated in Table 5.

To a Frame shape $\pi=\prod_{m \mid h} m^{\chi_{m}}$ one can associate a modular function [Kon]. Let

$$
\eta(\tau)=q^{1 / 24} \prod_{n=1}^{\infty}\left(1-q^{n}\right), \quad q=e^{2 \pi i \tau}, \tau \in \mathbb{H}
$$

be the Dedekind eta function. Then define

$$
\eta_{\pi}(\tau)=\prod_{m \mid h} \eta(m \tau)^{\chi_{m}}
$$

Let $\pi$ be a self-dual Frame shape of $\cdot 0$. By [Kon], $\eta_{\pi}$ is a modular function for a discrete subgroup $\Gamma^{\prime}$ of $S L(2, \mathbb{R})$ containing $\Gamma_{0}(h)$. The genus of $\Gamma^{\prime}$ is zero and $\eta_{\pi}$ is a generator of the function field of $\Gamma^{\prime}$. The groups $\Gamma^{\prime}$ corresponding to the Frame shapes of Table 5 are listed in $[\mathrm{CN}]$. 


\section{References}

[A1] Arnold, V. I., Normal forms of functions in neighbourhoods of degenerate critical points, Uspekhi Mat. Nauk, 29 (1974), 11-49 (Engl. translation in Russ. Math. Surv., 29 (1974), 19-48).

[A2] - Local normal forms of functions, Invent. Math., 35 (1976), 87-109.

[ATL] Conway, J. H., Curtis, R. T., Norton, S. P., Parker, R. A. and Wilson, R. A., ATLAS of Finite Groups, Oxford Univ. Press, London, New York, 1985.

[B] Bourbaki, N., Groupes et algèbres de Lie, Chapitres 4, 5 et 6. Hermann, Paris, 1968.

[CN] Conway, J. H. and Norton, S. P., Monstrous moonshine. Bull. Lond. Math. Soc., 11 (1979), 308-339.

[D1] Dolgachev, I. V., Quotient-conical singularities on complex surfaces, Funkt. Anal. Jego Prilozh., 8 (1974), 75-76 (Engl. translation in Funct. Anal. Appl., 8 (1974), 160-161.)

[D2] - Automorphic forms and weighted homogeneous singularities, Funkt. Anal. Jego Prilozh., 9 (1975), 67-68 (Engl. translation in Funct. Anal. Appl., 9 (1975), 149-151).

[D3] - Automorphic forms and weighted homogeneous equations, Preprint.

[D4] - On the link space of a Gorenstein quasihomogeneous surface singularity, Math. Ann., 265 (1983), 529-540.

[D5] - Mirror symmetry for lattice polarized K3 surfaces, J. Math. Sci., 81 (1996), 2599-2630.

[E1] Ebeling, W., The Milnor lattices of the elliptic hypersurface singularities, Proc. London Math. Soc. (3), 53 (1986), 85-111.

[E2] - The Monodromy Groups of Isolated Singularities of Complete Intersections, Lect. Notes in Math., 1293, Springer-Verlag, Berlin etc., 1987.

[E3] - Lattices and Codes, Vieweg, Wiesbaden, 1994.

[E4] - Strange duality, mirror symmetry, and the Leech lattice, In: Singularity Theory, Proceedings of the European Singularities Conference, Liverpool, 1996 (J. W. Bruce and D. Mond, eds.), Cambridge University Press, Cambridge, 1999, pp. 55-77.

[E5] - Strange duality and polar duality, J. London Math. Soc., 61 (2000), 823-834.

[E6] - Poincaré series and monodromy of a two-dimensional quasihomogeneous hypersurface singularity, Manuscripta Math., 107 (2002), 271-282.

[EW] Ebeling, W. and Wall, C. T. C., Kodaira singularities and an extension of Arnold's strange duality, Compositio Math., 56 (1985), 3-77.

[HM] Hirzebruch, F. and Mayer, K. H., $O(n)$-Mannigfaltigkeiten, exotische Sphären und Singularitäten, Lect. Notes in Math., 57, Springer-Verlag, Berlin etc., 1968.

[Kon] Kondo, T., The automorphism group of Leech lattice and elliptic modular functions, J. Math. Soc. Japan, 37 (1985), 337-361.

[La] Laufer, H., On minimally elliptic singularities, Amer. J. Math., 99 (1977), 1257-1295.

[Lo] Looijenga, E., The smoothing components of a triangle singularity. II, Math. Ann., 269 (1984), 357-387.

[N] Nikulin, V. V., Integral symmetric bilinear forms and some of their applications, Izv. Akad. Nauk. SSSR Ser. Mat., 43 (1979), 111-177 (Engl. translation in Math. USSR Izv., 14 (1980), 103-167).

[P1] Pinkham, H., Deformations of algebraic varieties with $G_{m}$ action, Astérisque, 20 (1974).

[P2] $\stackrel{-}{\longrightarrow}$, Normal surface singularities with $\mathbb{C}^{*}$ action, Math. Ann., 227 (1977), 183193.

[P3] - Singularités exceptionelles, la dualité étrange d'Arnold et les surfaces K-3, C. R. Acad. Sc. Paris, Série A, 284 (1977), 615-618.

[P4] - Deformations of normal surface singularities with $\mathbb{C}^{*}$ action, Math. Ann., 232 (1978), 65-84. 
[Sa1] Saito, K., On a duality of characteristic polynomials for regular systems of weights, Preprint, RIMS, Kyoto University, 1994.

[Sa2] - Duality for regular systems of weights, a précis, In: Topological Field Theory, Primitive Forms and Related Topics (M. Kashiwara, A. Matsuo, K. Saito and I. Satake, eds.), Progr. Math., 160, Birkhäuser, Boston Basel Berlin, 1998, pp. 379-426.

[Sh] Sherbak, I. G., Algebras of automorphic forms with three generators, Funkt. Anal. Jego Prilozh., 12 (1978), 93-94 (Engl. translation in Funct. Anal. Appl., 12 (1978), 156-158).

[VD] Van Dyke, F., Generators and relations for finitely generated graded normal rings of dimension two, Illinois J. Math., 32 (1988), 115-150.

[Wag1] Wagreich, P., Algebras of automorphic forms with few generators, Trans. AMS, 262 (1980), 367-389.

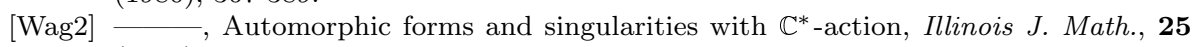
(1981), 359-382.

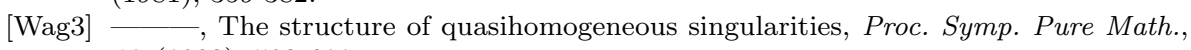
40 (1983), 593-611.

[Wal1] Wall, C. T. C., Classification of unimodal isolated singularities of complete intersections, Proc. Symp. Pure Math., 40 (1983), 625-640.

[Wal2] $\longrightarrow$, Notes on the classification of singularities, Proc. London Math. Soc., 48 (1984), 461-513.

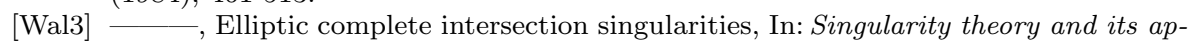
plications, Part I, Warwick 1989 (D. Mond and J. Montaldi, eds.), Lecture Notes in Math., 1462, Springer, Berlin etc., 1991, pp. 340-372. 